\title{
Stochastic Traffic and Connectivity Dynamics for Vehicular Ad-Hoc Networks in Signalized Road Systems
}

\author{
Ivan Wang-Hei Ho ${ }^{* \dagger}$, Kin K. Leung ${ }^{*}$, John W. Polak ${ }^{\dagger}$ \\ *Department of Electrical and Electronic Engineering, Imperial College London \\ ${ }^{\dagger}$ Centre for Transport Studies, Imperial College London \\ \{wh.ho, kin.leung, j.polak\}@imperial.ac.uk
}

\begin{abstract}
In the design and planning of vehicular ad-hoc networks, road-side infrastructure nodes are commonly used to improve the overall connectivity and communication capability of the networks, however, to determine the locations to install the infrastructure nodes for optimal performance based on the ever-changing density and connectivity dynamics of moving vehicles remains to be a challenging issue. In this paper, we introduce a stochastic traffic model to capture the space and time dynamics of vehicles in signalized urban road systems to identify poorly-connected regions for infrastructure node placements. To closely approximate the practical road conditions, we propose a density-dependent velocity profile to approximate vehicle interactions and capture platoons formation and dissipation at traffic signals. Numerical results are presented to evaluate the stochastic traffic model. In general, we show that the fluid model can adequately describe the mean behavior of the traffic stream, while the stochastic model can approximate the probability distribution well even when vehicles interact with each other as their movement is controlled by traffic lights. With the understandings of the vehicular density dynamics from the proposed model, we illustrate that connectivity dynamics of vehicles can be determined accordingly and consequent system engineering and planning issues such as the identification of poorlyconnected regions in the network for road-side infrastructure node placements can be carried out.
\end{abstract}

\section{INTRODUCTION}

In a Vehicular Ad-hoc Network (VANET), vehicles communicate with each other and with road-side infrastructure nodes. One of the major functions of infrastructure nodes is to fill the communication gaps between moving vehicles so as to improve connectivity in the network. However, the number of road-side infrastructure nodes that can be installed is limited primarily due to the cost. Therefore, to identify the right places for infrastructure nodes to optimize the network performance is of critical importance.

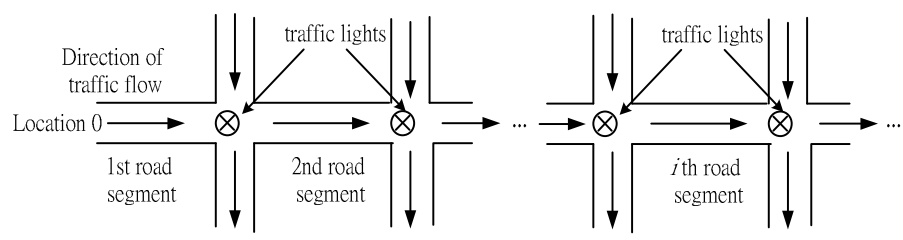

Figure 1. The road configuration considered in this paper.

We introduced in [1] a stochastic traffic model to capture the spatial and temporal details, accuracy of vehicle propagation along an urban route as illustrated in Figure 1, from which we can deduce the density and connectivity

The work reported in this paper forms part of the MESSAGE project. MESSAGE is a threeyear research project which started in October 2006 and is funded jointly by the UK Engineering and Physical Sciences Research Council and the UK Department for Transport. The project also has the support of nineteen non-academic organizations from public sector transport operations, commercial equipment providers, systems integrators and technology suppliers. More information is available from the web site www.message-project.org. The views expressed in this paper are those of the authors and do not represent the view of the Department for Transport or any of the non-academic partners of the MESSAGE project. dynamics of vehicles. The stochastic traffic model is a composite of the deterministic fluid dynamic model and the stochastic model. The densities of the mean or expected number of vehicles and the mean flow rate of the traffic stream are described by or readily computable from the conservation equations of fluid dynamics, which is in the form of a partial differential equation (PDE) as

$$
\frac{\partial n(x, t)}{\partial t}+\frac{\partial[n(x, t) v(x, t)]}{\partial x}=c^{+}(x, t)-c^{-}(x, t),
$$

where $n(x, t)$ and $v(x, t)$ are respectively the vehicular density and velocity in location $(0, x]$ at time $t, c^{+}(x, t)$, and $c^{-}(x, t)$ are the rate densities of the number of vehicles arriving to and departing from the route in location $(0, x]$ during time interval $(-\infty, t]$, respectively. We use the stochastic model to capture the randomness of individual vehicle and provide the probability distribution. When we consider vehicle arrivals as a Poisson process, the stochastic model becomes a special version of the Poisson Arrival Location Model (PALM) introduced in [2]. The actual number of vehicles in a given road section at a certain time instance thus has Poisson distribution according to previous PALM results in [3, 4] given that the arrival of vehicles follow a non-homogeneous Poisson process.

In this paper, we propose a density dependent velocity profile and an iterative algorithm to approximate vehicle interactions and shockwave propagation at traffic signals. We evaluate the quality of the stochastic traffic model with such approximation through extensive simulations. In essence, we show that the extra randomness inherited from vehicular interactions and traffic signals tends to be ignored by the system and the Poisson property of the model is still significant under such approximation of vehicle interactions. In addition, the solutions from the fluid model provide reasonable estimates for the mean number of vehicles in a given region, and the formation and dissipation of vehicular platoons at traffic signals are well accommodated by the density-dependent velocity profile given the signaling inputs. With the knowledge of the vehicular density dynamics from the proposed model, we illustrate that system engineering and network planning issues can be carried out. For instance, we show how the model can be used to identify the operating point for minimal transport (vehicle) congestion, and how it can be applied to identify regions with poor connectivity at such an operating point for the placement of road-side communication nodes to boost the overall communication connectivity.

\section{APPROXIMATION OF VEHICLE INTERACTIONS}

We can actually capture the propagation of shockwave through the fundamental relationship between vehicular density and velocity. In the followings, we propose the front-densitydependent velocity field based on the Greenshield's model [5] for such propose:

$$
v(x, t)=v_{f}\left(1-\frac{n(x+\Delta x, t)}{k_{j}}\right),
$$



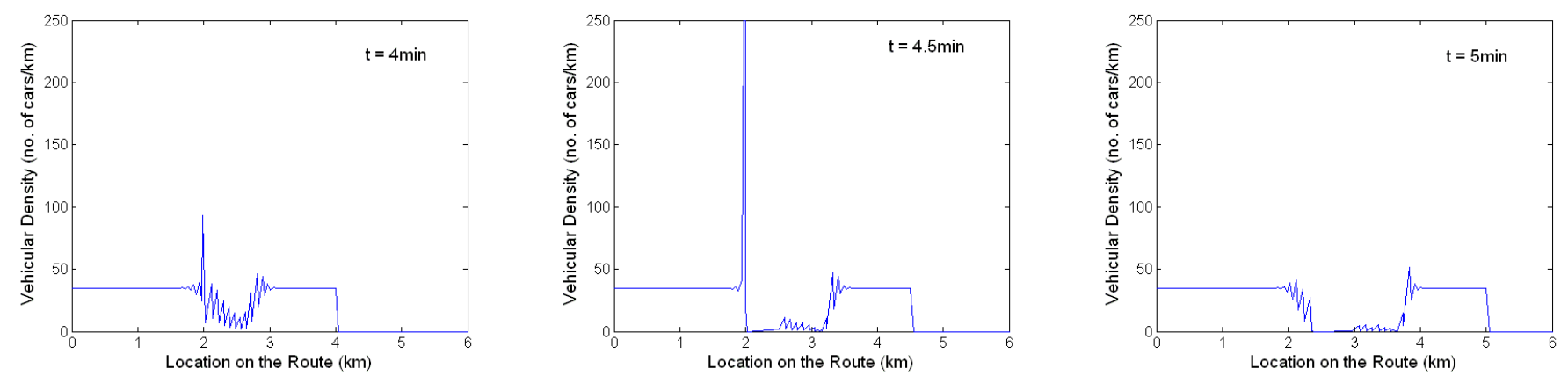

Figure 2. Platoon formation and dissipation at time instances 4, 4.5, and $5 \mathrm{~min}$ as described by the fluid dynamic model.

where $v_{f}$ represents the mean free speed and $k_{j}$ denotes the jamming density. If we define $l_{c}$ as the average space occupied by a car at stationary, then $k_{j}=1 / l_{c}$.

Eq. (2) illustrates our front-density-dependent velocity profile, instead of having the velocity at certain location $x$ as a function of density at location $x$, we have it as a function of density $\Delta x$ in front, the velocity decreases as the density in front increases. It is analogous to the car-following mechanism in transport studies, when the vehicular density in front becomes high (i.e., cars in front decelerate), we should decelerate as well. Such general traffic flow model is applicable to most of the urban route scenarios, no matter there are slowing down, stopping, start moving of vehicles or not.

With the front-density-dependent velocity profile, we can approximate the interactions between vehicles in the fluid dynamic model iteratively, the algorithm is illustrated in the pseudo code in Algorithm 1. The general idea is, initially, we assume there are no vehicles on the road, i.e., $n(x, 0)=0$ for all $x$ belongs to $X$, where $X$ is the location space. Therefore, the initial velocity will be the mean free speed $v_{f}$ according to (2). Based on these initial conditions, we solve the differential equations in the fluid dynamic model for the vehicular density along the route. We can then compute the new velocity profile for the next time slot from the vehicular density profile according to (2), and so on. We perform this algorithm iteratively until we reach some defined end time of the system.

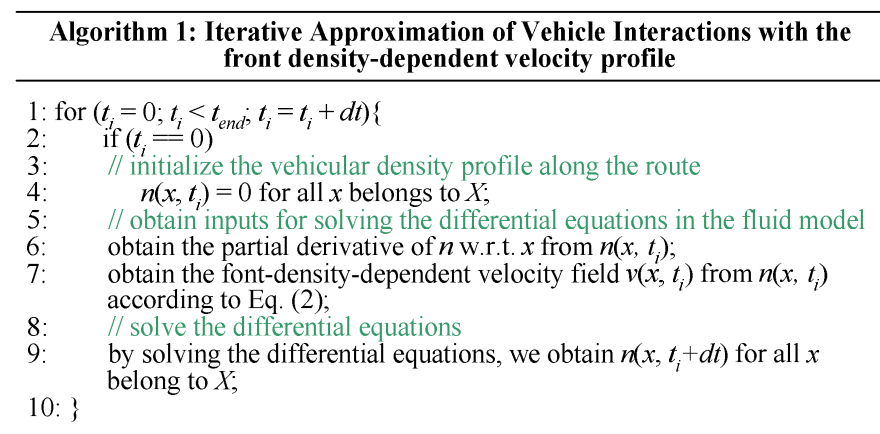

\section{NUMERICAL ANALYSIS}

In this section, we present numerical results to illustrate the space and time dynamics captured by the fluid dynamic model and the Poisson property captured by the stochastic model in a signalized urban route. Specifically, we evaluate the quality of the stochastic traffic model with the iterative approximation algorithm in terms of two metrics: i) the mean values computed from the fluid dynamic model; and ii) the distributional property from the stochastic model.

\section{A. Evaluation of the Stochastic Traffic Model with Approximation of Vehicle Interactions}

First of all, let us consider a simple stop-and-go motion example to evaluate $\mathrm{i}$ and ii above. In this example, we assume there are no cars joining and leaving the urban route at junctions, cars only arrive at location 0 at a constant rate (denoted by $\alpha$ ) of 30 cars $/ \mathrm{min}$, with the mean free speed, $v_{f}=1$ $\mathrm{km} / \mathrm{min}$, using the front-density-dependent velocity profile with $\Delta x=0.02 \mathrm{~km}$ as described in (2) unless otherwise specified. Assume the space occupied by a car at stationary as $4 \mathrm{~m}$, so the jamming density $k_{j}=250 \mathrm{cars} / \mathrm{km}$.

Consider that there is a traffic signal at location $2 \mathrm{~km}$, which stops vehicles from time 4 to $4.5 \mathrm{~min}$ for $30 \mathrm{sec}$, we allow a further $0.012 \mathrm{~km}$ distance behind the traffic signal as the length of the junction. Thus, when $4 \leq t<4.5, v=0$ if $2 \leq x$ $<2.012$ as shown in (3). In addition, we also include extra 0.02 $\mathrm{km}$ in front and behind the zero-velocity region so as to ensure that the partial derivative of $v$ w.r.t. $x$ is finite. For other regions during the stopping period, the front-density-dependent velocity field $v$ applies.

$$
v(x, t)=\left\{\begin{array}{cc}
v & \text { if } x<1.98 \\
(v / 0.02) *(2-x) & \text { if } 1.98 \leq x<2 \\
0 & \text { if } 2 \leq x<2.012 \\
(v / 0.02) *(x-2.02) & \text { if } 2.012 \leq x<2.032 \\
v & \text { if } \mathrm{x} \geq 2.032
\end{array}\right.
$$

When $\mathrm{t} \geq 4.5$, we instantaneously set the velocity in the junction $(2,2.012]$ to be the mean free speed $v_{f}$ (for a very short period of time) to represent that the first car in the queue move down the road with the highest speed when the signal turns green. The following cars then move according to the frontdensity-dependent velocity field. To model the stopping motion of vehicles more rigorously, we introduce an amber signal period for $3.95 \leq t<4$, such that the velocity within the junction region $(2,2.012]$ gradually decreases to zero at $t=4$ $\min$. So, for $t<3.95$, the system is in equilibrium state.

Figure 2 depicts the evolution of vehicular density along the signalized route as represented by the fluid model. From which we can see that the density pulse starts growing at $4 \mathrm{~min}$ due to the amber signal, and the jamming density pulse established and propagated backwards at $4.5 \mathrm{~min}$ due to the red signal. And lastly at $5 \mathrm{~min}$, the density pulse was dissipated by the green signal. Our results verify that the modeling of traffic signals and the platoon formation and dissipation process are achievable in the fluid model through the front-densitydependent velocity profile and additional signaling information.

Table 1. Comparison of the mean number of vehicles in the road region $(1.5,2]$ between the fluid model and simulation results.

\begin{tabular}{|c||c|c|c|c|}
\hline $\begin{array}{c}\text { Time instance } \\
(\mathrm{min})\end{array}$ & $\begin{array}{c}\text { Fluid } \\
\text { (\# of cars) }\end{array}$ & $\begin{array}{c}\text { Simulation } \\
\text { (\# of cars) }\end{array}$ & Error (\%) & D-statistic \\
\hline \hline 3.9 & 16.3874 & 15.3554 & 6.30 & 0.1581 \\
\hline 4 & 16.9526 & 16.8446 & 0.64 & 0.1527 \\
\hline 4.2 & 24.1911 & 22.6240 & 6.48 & 0.1331 \\
\hline 4.5 & 30.9754 & 31.2382 & 0.85 & 0.1197 \\
\hline 5 & 16.5939 & 15.3282 & 7.63 & 0.1638 \\
\hline
\end{tabular}




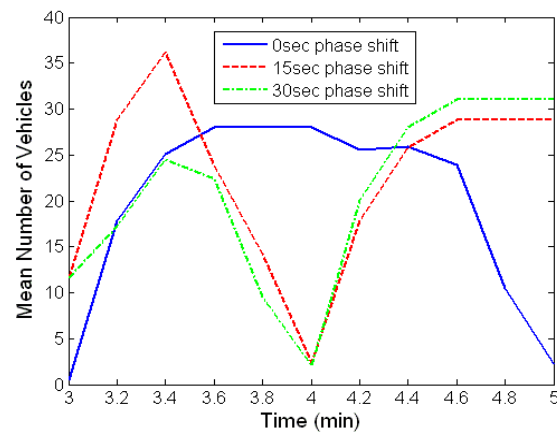

(a)

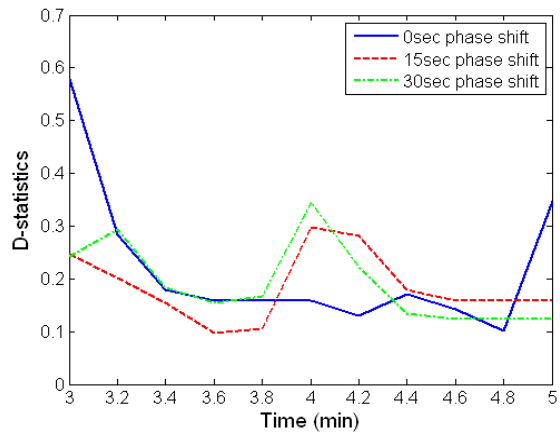

(b)

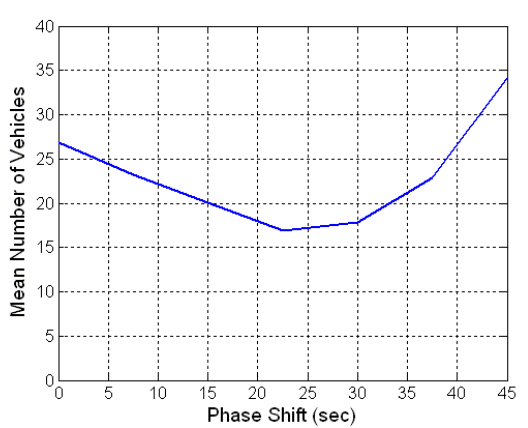

(c)

Figure 3. a) The mean number of vehicles in the road region $(2,2.5$ ] and b) the corresponding D-statistics against time with different phase shift between the two signals; and c) the space-time averaged number of vehicles in the road region $(2,2.5]$ from 3.4 to 4.4 min against phase shift.

To evaluate the expected values computed from the fluid dynamic model, we compare the solutions to the results from simulation. In the simulation, we have the same set of parameters described above including the vehicle arrival rate, velocity profile, and signaling inputs. We run the simulation for 5000 times with different random seeds, and record the number of vehicles in a given region at a given time. Thus, we have 5000 samples from the simulation for the mean and distribution evaluation. Specifically, we compare the mean number of vehicles in the road region $(1.5,2]$ at time instances $3.9,4,4.2,4.5$, and $5 \mathrm{~min}$. Table 1 compares the mean number of vehicles in the region $(1.5,2]$ on the urban signalized route at several time instances, from which we can see that the deviations between the solutions from the differential equations and simulation results are less than $7.7 \%$, and at time instances 4 and $4.5 \mathrm{~min}$, we can even achieve an error less than $1 \%$.

To evaluate if the distributions of the number of vehicles from simulations can be approximated with a Poisson distribution, we conduct the Kolmogorov-Smirnov test (K-S test) on their CDFs. As we aim to approximate the empirical CDF by certain hypothesized CDF (and not to prove that they are identical), we only examine the D-statistic value. If the value is small, it indicates that the empirical CDF can be approximated by the hypothesized CDF. In our case, the hypothesized CDF will be the CDF of Poisson distribution with parameter as the mean of the simulated results.

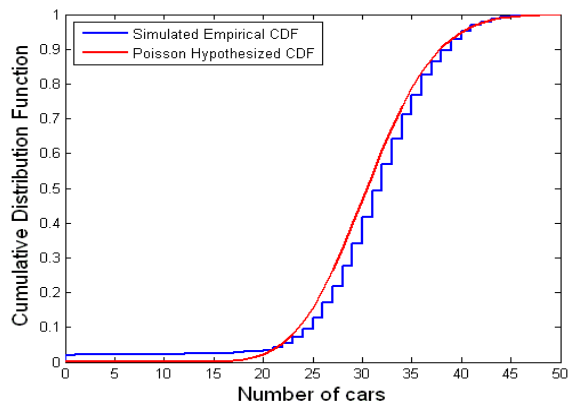

Figure 4. The empirical CDF and hypothesized CDF at time instance $4.5 \mathrm{~min}$.

We treat the case with deterministic velocity profile $(v=1$ $\mathrm{km} / \mathrm{min}$ ) as the benchmark for this goodness-of-fit study, which we found its D-statistic is about 0.1 . With regard to Table 1, the D-statistics of the simulation results remains small (at about 0.14 on average) for all time instances, while Figure 4 plots the empirical CDF of the number of vehicles within the road region $(1.5,2]$ at time instance $4.5 \mathrm{~min}$ for the 5000 simulation trials and the Poisson hypothesized CDF. They appear to be close to each other, and it is interesting to observe that the extra randomness that is brought upon by the densitydependent velocity profile and traffic signals does not seem to be destroying the Poisson property. For the stochastic independence, we found that given the arrival rate is not too high (e.g. $\leq 30 \mathrm{cars} / \mathrm{min})$, the degree of dependency between the number vehicles in two non-overlapping regions is low.

\section{B. Evaluation with Cascaded Traffic Signals}

We now evaluate the robustness of our model with more complicated traffic signal systems, and demonstrate the potential of our model for modeling vehicular density in urban routes with the ramifications of consecutive traffic signals for system engineering and planning in both the transport and communication networks.

Figure 5 depicts the street configuration for simulations. We consider two traffic signals that are $500 \mathrm{~m}$ apart, located at the 2 and $2.5 \mathrm{~km}$ locations respectively. Both of them have a 1 min cycle time, with $30 \mathrm{sec}$ green, $3 \mathrm{sec}$ amber, and $27 \mathrm{sec}$ red signal periods. The velocity profile is modeled in the same way as in the previous example according to the signal cycle. Assuming that cars only enter the route at location 0 with a rate of $30 \mathrm{cars} / \mathrm{min}$ and no cars join or depart at junctions in this example, we explore how the number of vehicles in the road segment $(2,2.5]$ varies with the phase shift between the two signals. We consider three cases here, they are respectively 1) the first and second signals are in phase; 2) the first signal is 15 sec lag behind the second one; and 3) the first signal is $30 \mathrm{sec}$ lag behind the second one.

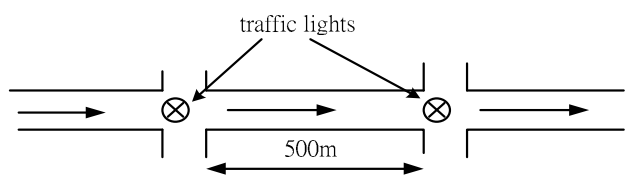

Figure 5. Street configuration test network.

Figure 3 plots the simulation results of a) the mean number of vehicles in the road region $(2,2.5]$ and $b)$ the corresponding D-statistics during the time interval $(3,5]$ for the three cases. First, we can see from Figure $3 b$ that the D-statistics remains small (at about 0.15) even with more complicated and oscillating traffic signal systems, except when the mean number of vehicles is small

Second, we can see that the signaling system plays an important role in distributing vehicles in both space and time dynamics. For example, it appears in Figure $3 \mathrm{a}$ that the signaling combination with no phase shift distributes cars more evenly during the time interval $(3.4,4.6]$ than the other two combinations with phase shift. Therefore, such observation motivates the future research direction of optimizing node connectivity in signalized roads by controlling the signaling system. For instance, we can have the signaling cycles or phase shift between consecutive traffic signals change according to vehicular density, in order to maintain a sufficient density level on each road segment for better connectivity in the communication network. 
On the other hand, from the transport network point of view, we can control the traffic load on a road segment in order to reduce transport congestions. Figure $3 \mathrm{c}$ shows how the mean number vehicles in the road region $(2,2.5]$ within the time interval $(3.4,4.4]$ varies with the phase shift between the two traffic signals, which appears that the minimal traffic congestion can be achieved if we set the phase shift to be about $22.5 \mathrm{sec}$. Therefore, through the stochastic traffic model, we can identify the operating point for minimal transport congestion, and at such operating point, our model can be further applied to identify regions with poor connectivity for the placement of road-side infrastructure nodes to boost the overall communication connectivity, which will be discussed in the next section.

\section{INFRASTRUCTURE NODE PLACEMENT}

We define $N\left(x_{a}, x_{b}, t\right)=N\left(x_{b}, t\right)-N\left(x_{a}, t\right)$, where $x_{b}>x_{a}$, as the number of vehicles in the region $\left(x_{a}, x_{b}\right]$ at time $t$. According to the stochastic model and previous performance evaluations, the actual number of vehicles distributed within a road region is Poisson distributed or approximately Poisson distributed even if we introduce vehicular interactions. Hence, the probability that there are at least $k$ vehicles located within a road region at time $t$ is given by

$$
P\left(N\left(x_{a}, x_{b}, t\right) \geq k\right)=1-\sum_{j=0}^{k-1} \frac{E\left[N\left(x_{a}, x_{b}, t\right)\right]^{j}}{j !} e^{-E\left[N\left(x_{a}, x_{b}, t\right)\right]}
$$

where $E\left[N\left(x_{a}, x_{b}, t\right)\right]=\int_{x_{a}}^{x_{b}} n(x, t) d x$, and the vehicular density profile $n(x, t)$ is computed from the fluid model. Based on (4) and the density profile $n(x, t)$, we have determined the general probability that the network in a road segment of length $L$ is connected at time $t$ as

$$
P(\text { net con })=\exp \left(-\int_{0}^{L-r} e^{-E[N(x+\Delta x, x+r, t)]} n(x, t) d x\right) .
$$

The proof of (5) is omitted for brevity but can be found in [1].

With the probability in (5), we can characterize the degree of connectivity in a road segment, and identify road segments that are likely to be disconnected in the network. However, the range of a road segment is still too large for us to determine the optimal position for infrastructure node placement. To further reduce the potential range, we can examine the time-averaged probability $P(N(x, x+r)>k)$ based on (4) for all $x$ belong to the road segment, the regions with low probability denote the desired locations for infrastructure nodes. It is illustrated below with an example.

As we have discussed in the previous section, we can identify poorly connected regions at the operating point of minimal transport congestion for the placement of road-side infrastructure nodes in order to optimize both the transport and communication networks. Let us consider the scenario in Section III.B again as an illustrative example. According to Figure $3 \mathrm{c}$, the minimal traffic congestion can be achieved if we set the phase shift to be $22.5 \mathrm{sec}$. At this operating point, given the communication range $r=100 \mathrm{~m}$ and a location point $x$, we can compute the time averaged probability of having at least $k$ vehicles in the region $(x, x+r]$ according to (4) so as to characterize the degree of connectivity of a specific location point with regard to the mean density information from the fluid model. Figure 6 plots the time averaged probability of having at least five forward neighbors as a function of the location space, from which we can identify the region $(2.05$, 2.28 ] is relatively poorly connected in the road segment (below a probability threshold of 0.25 ). Assuming that infrastructure nodes have the same transmission range as mobile nodes (100 $\mathrm{m})$, we place three infrastructure nodes at locations 2.1, 2.2 and $2.3 \mathrm{~km}$ to boost the connectivity of the network. The probability after the introduction of the three infrastructure nodes is shown by the red-dashed line in the figure, more number of infrastructure nodes can be added in the region to further raise the connectivity. Such optimization problems for both the transport and communication networks will serve as an extension for the traffic model, and the evaluation of their performance will be explored in the future.

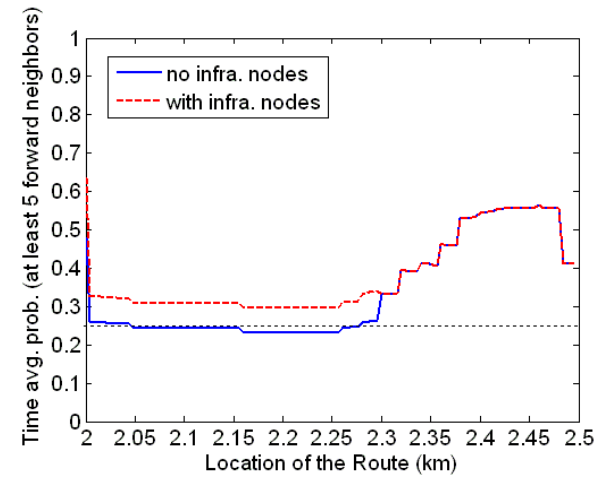

Figure 6. Connectivity as a function of location in road region $(2,2.5]$ over time interval $(3,5]$ in the two-traffic-light scenario, phase shift $=22.5 \mathrm{sec}$, transmission range $=100 \mathrm{~m}$, arrival rate $=30 \mathrm{cars} / \mathrm{min}$.

\section{CONCLUSIONS}

In this paper, we have presented approaches of modeling shockwave propagation with the velocity profile in the stochastic traffic model. Specifically, to closely approximate the practical road conditions, we have proposed the frontdensity-dependent velocity profile for approximating vehicle interactions in the model. With additional signaling inputs, it automatically captures the shock wave propagation of vehicles' stop-and-go motions at traffic signals. Numerical results were provided to evaluate the quality of the stochastic traffic model with approximation of vehicular interactions in terms of the expected value and distribution of its solution. In essence, we have showed that the fluid dynamic model well capture the evolution of the traffic stream, while the Poisson distributional conclusion of the stochastic model is still a good approximation for the distribution of the actual number of vehicles even when vehicles interact with each other as their movement is controlled by traffic signals

With the knowledge of the vehicular density dynamics from the stochastic traffic model, we have demonstrated its applicability on identifying regions with poor connectivity for road-side infrastructure node placements. In general, we have showed that the stochastic traffic model can serve as a useful tool for system engineering and planning for both communication networks and transport systems.

\section{REFERENCES}

[1] Ivan W. H. Ho, K. K. Leung, J. W. Polak, "Connectivity Dynamics for Vehicular Ad-hoc Networks in Signalized Road Systems," the 21st International Teletraffic Congress (ITC), Sept. 2009.

[2] W. A. Massey and W. Whitt, "Network of Infinite Server Queues with Nonstationary Poisson Input," Queuing Syst., vol.13, pp.183 - 250, 1993.

[3] W. A. Massey and W. Whitt, "A Stochastic Model to Capture Space and Time Dynamics in Wireless Communication Systems," Prob. in the Eng. \& Inf. Sci., vol.8, pp.541 - 569, 1994.

[4] K. Leung, W. A. Massey and W. Whitt, "Traffic Models for Wireless Communication Networks," IEEE J-SAC, vol. 12, no. 8, 1994.

[5] C. Khisty, B. Lall, Transportation Engineering, $2^{\text {nd }}$ ed. Prentice Hall, 1998. 